Acta vet. scand. $1976,17,343-353$.

From The State Veterinary Serum Laboratory, Copenhagen, Denmark.

\title{
A SEROLOGIC VARIANT OF MYCOPLASMA HYORHINIS RECOVERED FROM THE CONJUNCTIVA OF SWINE
}

\author{
By \\ N. F. Friis
}

FRIIS, N. F.: A serologic variant of Mycoplasma hyorhinis recovered from the conjunctiva of swine. Acta vet. scand. 1976, 17, 343 -353. - In an examination of conjunctival samples from 40 piglets for mycoplasmas, 17 isolates were obtained. Eight could be identified as Mycoplasma hyorhinis, three as Mycoplasma flocculare, and one as Acholeplasma sp. Five strains were not readily identifiable, but together with two previously recovered strains they were found to represent a distinct serogroup. All seven strains were glucose and phosphatase positive. Incubation in a $\mathrm{CO}_{2}$-enriched atmosphere led to enhancement of the growth on solid medium. The serogroup was serologically related to $M$. hyorhinis, but not to a number of other glucose fermenting species of mycoplasma, and it may therefore be regarded as a new subspecies of M. hyorhinis.

mycoplasma hyorhinis; mycoplasma flocculare; porcine conjunctival infection.

From an outbreak of conjunctivitis in a litter of piglets two strainis of mycoplasma were recovered. The initial growth in broth was rather slow and accompanied by acidification. Subcultivation on solid medium showed colonies of classical appearance. The isolates being not readily identifiable as Mycoplasma hyorhinis, and recovery of mycoplasmas from the conjunctival sac of swine seemingly not having been reported as yel, it was decided to examine a number of conjunctival samples for mycoplasmas.

The result of this work is reported in the present paper.

\section{MATERIAL AND METHODS}

Conjunctival mucous membranes were obtained from 40 dead piglets sent to this institute for diagnostic purposes. The animals 
were from four to 10 weeks old. The conjunctivae of one animal showed signs of inflammation, while the rest appeared normal.

\section{Cultivation}

The eyes of each animal were examined in pool. A piece of about $0.5 \times 1 \mathrm{~cm}$ was taken from the upper or lower conjunctival fornix of each eye and the material ground in a mortar. A $10 \%$ suspension in broth was prepared and used for inoculation of three different liquid media, 10-fold dilutions to $10^{-6}$ being made. One of the media (FF74) had been developed especially for Mycoplasma suipneumoniae (Friis 1975a). The other two media were of the Hayflick type (Friis 1975b), one (I) enriched with $0.1 \%$ arginine, $0.01 \%$ mucin, and $0.1 \%$ urea, the other (II) unenriched. To improve the bacteriostatic effect, $0.05 \mathrm{mg} / \mathrm{ml}$ cycloserine and $0.2 \mathrm{mg} / \mathrm{ml}$ vancomycin were added to Medium FF74. Cycloserine, $0.15 \mathrm{mg} / \mathrm{ml}$, was added to Medium I.

\section{Preliminary identification of isolates}

Recovered isolates were examined by the disc growth inhibition (DGI) test (Friis 1971) using antisera for known porcine mycoplasma species. Strains not identifiable by this procedure were examined more extensively as outlined below.

\section{Morphological and biochemical examination}

Medium FF74 was used for all subsequent examinations. The not readily identifiable strains were filtered through a $0.45 \mu \mathrm{m}$ filter and cloned three times on solid medium. Differentiation between the families Mycoplasmataceae and Acholeplasmataceae was made by means of the SPS (sodium-polyanethol-sulphonate) and digitonin tests (Friis $1975 \mathrm{~b}$ ) and by cultivation to $10^{-6}$ in liquid medium without serum. The possible reversion to a parent bacterium was examined in bacteriostatics-free medium. Three passages were made in liquid medium followed by cultivation on solid medium. The morphology of the isolates when cultivated in prefiltered liquid medium was studied by phase-contrast microscopy (magnification $1000 \times$ ). The optimum atmosphere for cultures on solid medium was examined in air, air $+5-10 \%$ $\mathrm{CO}_{2}$, and $\mathrm{N}_{2}+5-10 \% \mathrm{CO}_{2}$. A phosphatase test was performed in liquid medium with $0.05 \%$ phenolphthalein diphosphate added. After growth the cultures were alkalinised with $\mathrm{NaOH}$ 
to bring out the red color of possibly liberated phenolphthalein. The metabolic capacity was tested in liquid medium treated with glucose oxidase (Sigma) and enriched with PPLO serum fraction (Difco) instead of serum. The substrate to be tested, i.e. glucose, arginine, or urea, was added to a concentration of $0.1 \%$. Colorshift of phenol red in the medium was used as indication of metabolism.

\section{Serology}

The strains not readily identifiable (cf. above) were subjected to the disc growth inhibition (DGI) test and the metabolism inhibition (MI) test using rabbit antisera for the GDL (NCTC 10121) and BTS-7 (NCTC 10130) strains of Mycoplasma hyorhinis, and for a representative strain (Mp707) of the unknown isolates. In DGI, cultures were incubated in atmospheric air for one day at $29 \mathrm{C}$ followed by three days at $37 \mathrm{C}$. The GDL and BTS-7 strains and five cloned field strains of $M$. hyorhinis were included. MI was performed as a macrotest with 100-1000 color changing units of antigen (Friis 1971). In this test the GDL and BTS-7 strains were included.

Indirect immunofluorescence of unfixed colonies on agar using incident light (indirect IMF) was performed as described by Rosendal \& Black (1972). Antisera for the GDL and Mp707 strains were used in two-fold dilutions starting at 1/12.5. As in DGI, the GDL and BTS-7 strains and the five field strains of M. hyorhinis were included. FITC-labeled anti-rabbit immunoglobulin* was used. In addition, colonies of strain Mp707 were examined by indirect IMF against antisera ${ }^{\star *}$ for a large number of glucose positive mycoplasma species. Indirect hemagglutination (IHA) test with fresh, tanned sheep erythrocytes, carried out as described by Meyling (Mandrup et al. 1975), was used to compare strain Mp707 to the GDL strain. Mycoplasma bovirhinis (NCTC 10118) was incorporated for control. Antisera for each of the three strains were tested against antigens from all three strains. Double immunodiffusion (DID) between the Mp707 and

* Central Laboratory of the Netherlands Red Cross Blood Transfusion Service, Plesmanlaan 25, Amsterdam.

* Some of these antisera were obtained from Dr. S. Rosendal, FAO/ WHO International Reference Centre for Animal Mycoplasmas, Institute of Medical Microbiology, University of Aarhus, Denmark. 
GDL strains and M. bovirhinis was performed in PBS, $\mathrm{pH}$ 7.2, jellified with agarose ${ }^{\star}$. Polyethylene glycol 3000 was added to a concentration of $3.5 \%$. Wells measuring $8 \mathrm{~mm}$ were placed $5 \mathrm{~mm}$ apart. The mycoplasmas were cultivated in liquid medium, washed four times and concentrated 15 times by ultracentrifugation, and finally disrupted by 10 cycles of freezing and thawing. Each antigen was tested against all three antisera.

\section{RESULTS}

\section{Cultivation from the conjunctiva}

From the conjunctivae of the 40 dead piglets 17 isolates were obtained, all from conjunctivae without signs of inflammation. All the isolates caused yellow colorshift in the primary cultures. No growth of arginine or urea metabolising strains was noted. The preliminary identification of the isolates is given in Table 1 .

T a b le 1. Identification by disc growth inhibition of mycoplasmas recovered from the conjunctiva of piglets.

\begin{tabular}{cccccc}
\hline Number of animals & \multicolumn{4}{c}{ Number of isolates inhibited by antiserum for } \\
\cline { 2 - 6 } examined/positive & $\begin{array}{c}\text { M. hyo- } \\
\text { rhinis }\end{array}$ & $\begin{array}{c}\text { M. floc- } \\
\text { culare }\end{array}$ & Mp707 & $\begin{array}{c}\text { Achole- } \\
\text { plasma sp.* * }\end{array}$ & $\begin{array}{c}\text { Other myco- } \\
\text { plasmas }\end{array}$ \\
\hline $40 / 16^{\star}$ & 8 & 3 & 5 & 1 & 0 \\
\hline
\end{tabular}

* One animal harboured both M. hyorhinis and M. flocculare.

* * Not readily identifiable, but resistant to SPS and digitonin, and capable of growing at $22 \mathrm{C}$.

It appears that M. hyorhinis, M. flocculare, Acholeplasma sp., and the unknown group, represented by $\mathrm{Mp} 707$, were demonstrated, but not M. suipneumoniae. The strains of the unknown group showed growth in Medium FF74 and in Medium II.

Identification of strains related to Mp707

Cultural characteristics. Seven strains were studied, two of which were from the outbreak of conjunctivitis and five from the dead piglets. The strains originated from six herds situated in different parts of the country.

* Indubiose A 37, L'Industrie Biologique Francaise S.A., 92 Gennevilliers, France. 
On primary isolation in broth the growth of all strains was slow, with just a moderate yellow colorshift. After two-four pasisages in broth the speed and intensity of growth increased rapidly. After cloning all strains would form colonies of the typical "fried-egg" type when cultured for two-three days on solid medium. Incorporation of $5-10 \% \mathrm{CO}_{2}$ in a basal atmosphene of $\mathrm{N}_{2}$ or air greatly improved the growth on the agar plates. In the SPS and digitonin tests all the strains were sensiitive, with zones of inhibition of at least $5 \mathrm{~mm}$. In liquid medium without serum no evidence of growth was seen after incubation for one week. After three passages in bacteriostatics-free liquid media no reversion to a parent bacterium was observed, in that only typical mycoplasma-like colonies developed when the strains were transferred to solid medium. Phase-contrast microscopy of broth cultures revealed coccoid, filamentous, and pleomorphic

T able 2. Disc growth inhibition test on unknown mycoplasmas recovered from the conjunctiva of piglets, as compared to reference and field strains of M. hyorhinis.

\begin{tabular}{lccc}
\hline Antigen & \multicolumn{3}{c}{ Antiserum for } \\
\cline { 2 - 4 } & Mp707 & $\begin{array}{c}\text { M. hyorhinis } \\
\text { GDL }\end{array}$ & $\begin{array}{c}\text { M. hyorhinis } \\
\text { BTS-7 }\end{array}$ \\
\hline Unknown strains & & & \\
Mp707 & 7 & 0 & 0 \\
Mp708 & 5 & 0 & 0 \\
Mp443 & 2 & 0 & 0 \\
Mp451 & 3 & 0 & 0 \\
Mp456 & 2 & 0 & 0 \\
Mp457 & 4 & 0 & 0 \\
Mp478 & 2 & 0 & 0 \\
M. hyorhinis, ref. strains & & & \\
GDL (NCTC 10121) & 0 & 5 & 4 \\
BTS-7 (NCTC 10130) & 0 & 4 & 5 \\
M. hyorhinis, field strains & & & nd \\
M115 & 0 & 4 & nd \\
M117 & 0 & 3 & nd \\
M118 & 3 & 6 & nd \\
M119 & 0 & 4 & nd \\
M120 & 0 & 5 & \\
\hline
\end{tabular}

nd $=$ not done.

Figures refer to the term "relative reduction", i.e. the size of the zone with definite reduction of colonies, either in number or in size (Whittlestone, personal communication). 
elements. In the phosphatase test all the strains were clearly positive. All strains examined showed ability to degrade glucose ${ }^{\star}$, but not arginine or urea. Production of "film \& spot" was not examined in special media, but the phenomenon did not appear in the medium used.

Serological investigations. The results of DGI are shown in Table 2. All the unknown strains were inhibited by antiserum for the representative strain of the group (Mp707), while none were inhibited by antisera for the reference strains of M. hyorhinis (GDL and BTS-7). None of the reference strains and only one of the field strains (M118) of M. hyorhinis were inhibited by Mp707 antiserum.

T a b l e 3. Metabolism inhibition test on unknown mycoplasmas recovered from the conjunctiva of piglets, as compared to the reference strains of M. hyorhinis.

\begin{tabular}{lrcc}
\hline Antigen & \multicolumn{3}{c}{ Antiserum for } \\
\cline { 2 - 4 } & Mp707 & $\begin{array}{c}\text { M. hyorhinis } \\
\text { GDL }\end{array}$ & $\begin{array}{c}\text { M. hyorhinis } \\
\text { BTS-7 }\end{array}$ \\
\hline Unknown strains & & & \\
Mp707 & 640 & 80 & 20 \\
Mp708 & 320 & 80 & 20 \\
Mp443 & 320 & 40 & $<20$ \\
Mp451 & 320 & 80 & $<20$ \\
Mp456 & 2560 & 640 & 160 \\
Mp457 & 10240 & 160 & 160 \\
Mp478 & 320 & 80 & $<20$ \\
M. hyorhinis, ref. strains & & & 20 \\
GDL (NCTC 10121) & 640 & 5120 & 2560 \\
BTS-7 (NCTC 10130) & 640 & 40960 & 81920 \\
\hline
\end{tabular}

Figures indicate titres expressed as reciprocals of MI endpoint dilution.

In MI (Table 3) all the unknown strains were inhibited to significant titres by Mp707 antiserum, and also by antiserum for the GDL strain of M. hyorhinis, but to lower titres. Some were also inhibited by antiserum for the BTS-7 strain of M. hyorhinis.

* As also the glucose-free controls showed some degree of yellow colorshift, the breakdown of glucose was confirmed chemically. For this analysis the author is indebted to Dr. Conny Wolstrup of this institute. 
T a ble 4. Indirect immunofluorescence on colonies of unknown mycoplasmas recovered from the conjunctiva of piglets, as compared to the GDL reference strain and some field strains of M. hyorhinis.

\begin{tabular}{|c|c|c|c|c|}
\hline \multirow[t]{3}{*}{ Antigen } & \multicolumn{4}{|c|}{ Antiserum for } \\
\hline & \multicolumn{2}{|c|}{ Mp707 } & \multicolumn{2}{|c|}{ M. hyorhinis, GDL } \\
\hline & strong & moderate & strong & $\overline{\text { moderate }}$ \\
\hline \multicolumn{5}{|l|}{ Unknown strains } \\
\hline Mp707 & 800 & 800 & 12.5 & 50 \\
\hline Mp708 & 400 & 800 & 25 & 50 \\
\hline Mp443 & 200 & 400 & $<12.5$ & $<12.5$ \\
\hline Mp451 & 200 & 400 & 12.5 & 50 \\
\hline Mp456 & 400 & 800 & 25 & 50 \\
\hline $\mathrm{Mp} 457$ & 400 & $>1600$ & $<12.5$ & 25 \\
\hline $\operatorname{Mp} 478$ & 200 & 400 & $<12.5$ & $<12.5$ \\
\hline \multicolumn{5}{|l|}{ M. hyorhinis, ref. strain } \\
\hline GDL (NCTC 10121) & 12.5 & 100 & 200 & 200 \\
\hline \multicolumn{5}{|c|}{ M. hyorhinis, field strains } \\
\hline M115 & 50 & $>200$ & 400 & $>1600$ \\
\hline M117 & $<12.5$ & $<12.5$ & 100 & 200 \\
\hline M118 & 25 & 100 & 400 & 400 \\
\hline M119 & 100 & $>200$ & 400 & 800 \\
\hline M:120 & $<12.5$ & 100 & 200 & 400 \\
\hline
\end{tabular}

strong $=$ strong fluorescence.

moderate $=$ moderate, but still distinct fluorescence.

Figures indicate titres expressed as reciprocals of highest antiserum dilution giving fluorescence.

In indirect IMF tests (Table 4) all the unknown strains showed strong fluorescence to titres of 200 or higher with $\mathrm{Mp707}$ antiserum. With antiserum for the GDL strain four strains gave strong fluorescence to titres of 25 or 12.5 , one gave moderate fluorescence to a titre of 25 , and two did not react. The GDL strain gave strong fluorescence to a titre of 200 with homologous antiserum and to 12.5 with Mp707 antiserum. The five field strains of $\mathrm{M}$. hyorhinis showed strong fluorescence to a titre of 100 or above with GDL antiserum. Four of them reacted to lower titres with Mp707 antiserum. Examination of Mp707 by indirect IMF (not in table) turned out negative with dilutions $1 / 12.5$ and $1 / 25$ of antisera for the following glucose metabolising species of the genus Mycoplasma: Al Aubaidi L; M. anatis; M. bovirhinis; M. bovoculi; M. canis; M. capricolum; M. caviae; M. conjunctivae; M. cynos; M. dispar; M. edwardii; M. felis; M. fermentans; 
M. flocculare; M. gallinarum; M. gallisepticum, Group 7 (Leach); M. macullosum; M. molare; M. mycoides subspecies mycoides; M. mycoides subspecies capri; M. neurolyticum; M. ovipneumoniae; M. pneumoniae; M. pulmonis : M. putrefaciens; M. suipneumoniae.

Table 5. Double immunodiffusion with strain Mp707 of the unknown conjunctiva isolates, the GDL strain of M. hyorhinis, and M. bovirhinis.

\begin{tabular}{lccc}
\hline Antigen & \multicolumn{3}{c}{ Antiserum for } \\
\cline { 2 - 4 } & Mp707 & M. hyorhinis, GDL & M. bovirhinis \\
\hline $\begin{array}{l}\text { Mp707 } \\
\begin{array}{l}\text { M. hyorhinis } \\
\text { GDL (NCTC 10121) }\end{array}\end{array}$ & 3 & $1(2)$ & $0(1)$ \\
$\begin{array}{l}\text { M. bovirhinis } \\
\text { (NCTC 10118) }\end{array}$ & 0 & 3 & 0 \\
\hline
\end{tabular}

Figures indicate number of precipitation lines, in brackets if occurring irregularly by repetition of test.

In DID, strain Mp707 was compared to the GDL strain with M. bovirhinis included as a control (Table 5). Both Mp707 and GDL regularly gave three distinct lines of precipitation with homologous antiserum but only one or two on cross testing. An irregularly occurring line of precipitation was also observed on testing Mp707 against antiserum for M. bovirhinis.

T a b l e 6. Indirect hemagglutination with strain Mp707 of the unknown conjunctiva isolates, the GDL strain of M. hyorhinis, and M. bovirhinis.

\begin{tabular}{lccc}
\hline Antigen & \multicolumn{3}{c}{ Antiserum for } \\
\cline { 2 - 4 } & Mp707 & M. hyorhinis, GDL & M. bovirhinis \\
\hline $\begin{array}{l}\text { Mp707 } \\
\begin{array}{l}\text { M. hyorhinis } \\
\text { GDL (NCTC 10121) }\end{array}\end{array}$ & 100 & 100 & $<12.5$ \\
$\begin{array}{l}\text { M. bovirhinis } \\
\text { (NCTC 10118) }\end{array}$ & $<12.5$ & 400 & $<12.5$ \\
\hline
\end{tabular}

Figures indicate titres expressed as reciprocals of highest antiserum dilution giving agglutination. 
In IHA (Table 6) Mp707 and GDL both showed reaction to a titre of $\mathbf{4 0 0}$ with homologous antiserum and cross reaction to a titre of 100. A titre of 25 was recorded on testing M. bovirhinis against GDL antiserum.

\section{DISCUSSION}

Strains of M. hyorhinis, M. flocculare, and Acholeplasma sp. were identified among 17 isolates from the conjunctivae of $\mathbf{4 0}$ piglets. Five of the 17 strains, all showing the classical mycoplasma-like "fried-egg" colony morphology, were not readily identified. Having been found by DGI to be mutually related, these and two previously recorded strains were submitted to more detailed examination.

By phase-contrast microscopy all seven unknown strains showed pleomorphism and mycelial growth. Since they failed to revert to a parent bacterium after growth in bacteriostatios-free medium they were likely to be mycoplasmas. Showing no notable growth in serum-free medium and being sensitive to SPS and digitonin, the strains were referred to the family Mycoplasmataceae (Freundt et al. 1973).

All the strains being glucose and phosphatase positive, but arginine and urea negative, they obviously share the most important biochemical features with M. hyorhinis (Aluotto et al. 1970, Friis 1974).

Antigenically all seven unknown strains were found closely related by the classical DGI and MI tests (Tables 2 and 3 ). This finding was confirmed (Table 4) by indirect IMF technique applied to unfixed colonies on agar. This technique apparently being decisive for mycoplasma identification at species level (Freundt 1974) the seven strains obviously belong to one and the same species. The high titres obtained by indirect IMF with antiserum for the representative of the group (Mp707) indicate that they belong to one serogroup.

Examination of the relation of the new serogroup to established species of mycoplasma soon revealed antigenic relatedness to the porcine species M. hyorhinis, and efforts were therefore concentrated on the confirmation hereof. By DGI little cross reaction was found between the new serogroup and $M$. hyorhinis, none of the seven strains being inhibited by antisera for the two reference strains of M. hyorhinis (GDL and BTS-7) and just one (M118) of seven strains of M. hyorhinis being inhibited by anti- 
serum for the representative of the new serogroup (Mp707). On the other hand both MI and indirect IMF clearly revealed its relatedness to M. hyorhinis. Thus, in MI all the strains of the new serogroup were significantly inhibited by GDL antiserum, though invariably to lower titres than by Mp707 antiserum. Just four of the strains were inhibited by BTS-7 antiserum, and two of them to a titre of 20 only. Conversely, antiserum for Mp707 inhibited the growth of the reference strains of M. hyorhinis. The relatedness was further substantiated by indirect IMF testing of the seven strains of the new serogroup and seven strains of M. hyorhinis against Mp707 and GDL antisera. In this tesit it was noteworthy that in heterologous reactions in both groups there was a tendency for the range between strong and moderate fluorescence to be rather wide. By DID and IHA (Tables 5 and 6) the antigenic relationship of the new serogroup to $M$. hyorhinis was further corroborated. While a cross reaction in DID does not seem to be species specific (Kenny 1969, Rosendal 1975) for mycoplasma, there is little doubt that the relatively high heterologous titres obtained by IHA crosstesting of strains Mp707 and GDL indicate a relation at species level.

The negative outcome of indirect IMF on Mp707 with antisera for a number of glucose fermenting mycoplasma species indicate that the serogroup differs antigenically from these species.

From the investigations described in this paper it seems reasonable to conclude that the seven unknown isolates from conjunctivae of swine represent a distinct serogroup within the species Mycoplasma hyorhinis, perhaps as a new subspecies. Strain Mp707 has been chosen as the representative of the group. The results of MI may reflect a closer relationship of the serogroup to the reference strain GDL of M. hyorhinis than to the type strain BTS-7 of that species. Comparing the beneficial effect of $\mathrm{CO}_{2}$ on cultures on solid medium, which is a feature of all the members of the new serogroup, to the fact that there is a high content of carbonates in lachrymal fluid, one may venture the conjecture that the serogroup represents an undergroup of $M$. hyorhimis specially adapted to the conjunctival sac.

\section{REFERENGES}

Aluotto, Barbara B., Ruth G. Wittler, Carol O. Williams \& J. E. Faber: Standardized bacteriologic techniques for the characterization of mycoplasma species. Int. J. system. Bact. 1970, 20, 35-58. 
Freundt, E. A.: Practical aspects of serological identification. Inserm 1974, 33, 161-168.

Freundt, E. A., B. E. Andrews, H. Ern $\phi$, M. Kunze \& F. T. Black: The sensitivity of Mycoplasmatales to sodium-polyanethol-sulfonate and digitonin. Zbl. Bakt., I. Abt. Orig. A 1973, 225, 104-112.

Friis, N. F.: Mycoplasmas cultivated from the respiratory tract of Danish pigs. Acta vet. scand. 1971, 12, 69-79.

Friis, N.F.: Mycoplasmas in pigs with special regard to the respiratory tract. Thesis. DSR Forlag, Copenhagen 1974.

Friis, N. F.: Some recommendations concerning primary isolation of Mycoplasma suipneumoniae and Mycoplasma flocculare. Nord. Vet.-Med. 1975a, 27, 337-339.

Friis, N. F.: The SPS and digitonin tests applied to porcine mycoplasmas. Acta vet. scand. $1975 \mathrm{~b}, 16,474-476$.

Kenny, G. E.: Serological comparison of ten glycolytic mycoplasma species. J. Bact. 1969, 98, $1044-1055$.

Mandrup, M., N. F. Friis, A. Meyling \& J. H. Meding: Studies on the possible occurrence of mycoplasmas in boar semen. Nord. Vet.Med. 1975, 27, 557-561.

Rosendal, S.: Canine mycoplasmas: serological studies of type and reference strains with a proposal for the new species, Mycoplasma opalescens. Acta path. microbiol. scand. Sect. B 1975, $83,463-470$.

Rosendal, S. \& F. T. Black: Direct and indirect immunofluorescence of unfixed and fixed mycoplasma colonies. Acta path. microbiol. scand. Sect. B 1972, 80, 615-622.

\section{SAMMENDRAG}

En serologisk variant af Mycoplasma hyorhinis isoleret fra conjunctiva hos svin.

I en undersøgelse over forekomst af mykoplasmer i conjunctiva hos svin isoleredes 17 stammer fra $i$ alt 40 unders $\varnothing$ gte smågrise. Otte stammer kunne identificeres som Mycoplasma hyorhinis, 3 som Mycoplasma flocculare og 1 som Acholeplasma sp. Fem stammer lod sig ikke umiddelbart identificere, men sammen med 2 tidligere isolerede, lignende stammer påvistes de at repræsentere én og samme serogruppe. Alle 7 stammer fandtes glukose positive og fosfatase positive. Deres vækst på fast substrat forbedredes betydeligt ved at incorporere $\mathrm{CO}_{2} \mathrm{i}$ den omgivende atmosfære, hvad enten denne bestod af alm. luft eller kvælstof. Ved serologiske unders $\varnothing$ gelser påvistes serogruppen at være antigent beslægtet med M. hyorhinis, men ikke med andre glukoseforgærende mykoplasma arter. Derfor synes serogruppen at repræsentere en ny underart af $M$. hyorhinis.

(Received July 19, 1976).

Reprints may be requested from: N. F. Friis, The State Veterinary Serum Laboratory, Bülowsvej 27, DK-1870 Copenhagen V, Denmark. 